\title{
An ICME impact on the Martian hydrogen corona
}

\author{
Qi Xu, XiaoJun Xu*, Qing Chang, JiaYing Xu, Jing Wang, and YuDong Ye
}

State Key Laboratory of Lunar and Planetary Sciences, Macau University of Science and Technology, Macau 999078, China

\section{Key Points:}

- Mars encountered an ICME on 7 March 2015

- The pick-up $\mathrm{H}^{+}$flux in upstream solar wind was enhanced during the ICME event

- The penetrating $\mathrm{H}^{+}$flux in the Martian atmosphere also increased

Citation: Xu, Q., Xu, X. J., Chang, Q., Xu, J. Y., Wang, J., and Ye, Y. D. (2020). An ICME impact on the Martian hydrogen corona. Earth Planet. Phys., 4(1), 38-44. http://doi.org/10.26464/epp2020006

\begin{abstract}
The Martian hydrogen exosphere extends out of the bow shock, forming a "hydrogen corona". The solar wind interacts directly with the hydrogen corona. During an ICME event on 7 March 2015, the SWIA instrument onboard Mars Atmosphere and Volatile Evolution mission (MAVEN) observed that the pick-up $\mathrm{H}^{+}$fluxes in upstream solar wind were enhanced. Also increased were the penetrating $\mathrm{H}^{+}$fluxes in the Martian atmosphere. Quantitatively, these penetrating $\mathrm{H}^{+}$fluxes cannot increase by a factor of 5 simply due to a factor of 3 increase in the solar wind density, suggesting that the increased abundance of exospheric hydrogen upstream of the bow shock was a consequence of the passage of the ICME. A denser outer hydrogen corona at high altitudes suggests that the expansion of the neutral atmosphere was caused by the ICME. The excited and heated hydrogen exosphere probably indicates an elevated hydrogen escape rate during an ICME.
\end{abstract}

Keywords: Mars; hydrogen corona; ICME

\section{Introduction}

Mars is surrounded by an atomic hydrogen corona observable at the wavelength of Lyman- $\alpha$ (Anderson and Hord, 1971; Chaufray et al., 2008; Chaffin et al., 2015). The origin of exospheric hydrogen atoms is water vapor. Water vapor at the near-surface atmosphere can produce molecular hydrogen through photochemical reactions, and the $\mathrm{H}_{2}$ diffuses slowly from low altitudes to the ionosphere (McElroy and Donahue, 1972). The upward transport of molecular hydrogen is a stable process but on a shorter timescale (of weeks) the variation of water vapor at high altitudes could influence the exospheric $\mathrm{H}$ population, leading to seasonal variation of the Martian hydrogen corona (Bhattacharyya et al., 2015; Chaffin et al., 2017). In addition to the intrinsic atmospheric dynamics (Chaffin et al., 2017), the solar radiation inputs (Chaufray et al., 2015; Halekas, 2017) and solar wind (Bhattacharyya et al., 2015) may have an effect on the variability of the planet's $\mathrm{H}$ exosphere.

Interplanetary coronal mass ejections (ICMEs) are the manifestations of coronal mass ejections (CMEs) in interplanetary space, characterized by enhanced plasma density, lower plasma temperature, and stronger magnetic field with respect to ambient solar wind. Such space storms might have been more common in the early period of the solar system's history. Under the impact of

Correspondence to: X. J. Xu, xjxu@must.edu.mo

Received 11 OCT 2019; Accepted 17 JAN 2020.

Accepted article online 21 JAN 2020.

(C) 2020 by Earth and Planetary Physics.
ICMEs, plasma environment disturbances can be observed not only at Earth (Mannucci et al., 2005), but also at Venus (Xu Q et al., 2019) and Mars (Jakosky et al., 2015a). Study of ICME encounters with the Martian hydrogen corona can provide deeper understanding of the interactions between space weather events and weakly magnetized planets. During an ICME with increased solar wind proton flux, the ion escape rate at Mars increases, as evidenced by the enhancement of pick-up $\mathrm{O}^{+}$flux (Jakosky et al., 2015a; Curry et al., 2015). Different from heavy particles, the hydrogen loss to space is primarily by thermal (or Jeans) escape (Jakosky et al., 2018). To investigate the hydrogen escape rate at Mars during ICMEs, Mayyasi et al. (2018) studied the response of hydrogen Lyman- $\alpha$ emission to a space weather event. Based on a comparison of observations with simulation results, they suggested that the hydrogen abundance decreased while the temperature increased at the exobase, and the derived Jeans escape rate increased by a factor of 5 during the space weather event.

Because Mars lacks a global dipole magnetic field, the impact of an ICME on Mars is quite different from that on Earth (Curry et al., 2015). The Martian H corona extends outward more than 10 Mars radii, a distant region upstream of the bow shock (Chaffin et al., 2015). With the hydrogen corona exposed directly to the solar wind, charge exchange between solar wind protons and the neutral hydrogen atoms will produce new protons and new $\mathrm{H}$ atoms. The newly born $\mathrm{H}^{+}$can be picked up by the solar wind convection electric field and escape from the planet, while the newly born $\mathrm{H}$ atoms, without the resistance of an electromagnetic field, can penetrate into the atmosphere and collide with atmospheric 
particles, producing "penetrating $\mathrm{H}^{+"}$ (Halekas, 2017). Though UItraviolet (UV) radiation observations of the corona might be contaminated during solar storms, the properties of the hydrogen corona can also be evidenced by indirect observations, including pick-up $\mathrm{H}^{+}$(Rahmati et al., 2017, 2018) and penetrating $\mathrm{H}^{+}$(Halekas, 2017).

In this study, by using observations from the Solar Wind lon Analyzer (SWIA) (Halekas et al., 2015a) onboard the Mars Atmosphere and Volatile EvolutioN mission (MAVEN) (Jakosky et al., 2015b), we investigate comprehensively the responses of the Martian hydrogen corona to the ICME event on 7 March 2015.

\section{Instrument and Data}

MAVEN is an orbiter mission developed by NASA. The mission aims at understanding the relationship between atmospheric loss and climate change that occurred in the history of Mars, including a science objective to explore the interactions of the solar wind with the Martian upper atmosphere (Jakosky et al., 2015b). The SWIA carried by MAVEN measures ions in a broad $360^{\circ} \times 90^{\circ}$ field of view and across the energy range of $0.005-25 \mathrm{keV}$, divided into 96 energy steps (Halekas et al., 2015a). The Coarse product, in which anodes in the sunward direction are summed up and adjacent energy steps are summed over, presents a total $16 \times 4 \times 48$ array (16 anode bins labeled from $A 0$ to $A 15,4$ deflection bins labeled from D0 to D3, and 48 energy bins), while the timeordered angle-averaged (one-dimensional) ion energy spectra, as computed from Coarse ion distributions, contains a 48-element array of differential energy fluxes (Halekas et al., 2015a). The moments produced by SWIA reveal the basic properties of the solar wind (Halekas et al., 2015a). However, SWIA does not have a capability to discriminate different ion species (Halekas et al., 2015a). SWIA is mounted to a small platform at the corner of the spacecraft, with an axis pointing to the sun and an axis points toward the nadir for the majority of operational attitudes (Halekas et al., 2015a).

\section{Observations}

\subsection{Overview of the ICME on 7 March 2015}

Three main solar storms observed by MAVEN occurred in December 2014, March 2015, and September 2017. There was no dayside atmosphere observation during the first storm and no solar wind observation during the third one. A series of ICMEs happened in March 2015; the ICME event on 7 March 2015 has been reported previously (Jakosky et al., 2015a; Lee et al., 2017). This ICME erupted on 1 March and arrived at Mars on 7 March 2015, just before the arrival of the widely reported ICME of 8 March 2015 (e.g., Lee et al., 2017). Mars had experienced a short period in the quiet solar wind after the most recent previous ICME, encountered on 4 March (Jakosky et al., 2015a). Figure 1 shows the overview of this event based on the Mars-Solar-Orbital (MSO) coordinate system, where $+X$ points from Mars to the Sun along Mars-Sun line, the $+Y$ direction points oppositely to the planet's direction of motion, and the $Z$ axis remains perpendicular to the planet's orbital plane to complete the right-handed set; the origin is always at the geometrical center of Mars. Figure 1a displays the magnitude of the magnetic field from 12:00 UT on 6 March to 12:00 UT on 7 March. The vertical black lines mark the bow shock crossings in Orbits 837 and 840 . Vertical grey dash lines indicate the periapsis crossings. The strongest magnetic field appears in Orbit 840 . The second panel displays the three components of the magnetic field. In Figure 1c, solar wind velocity stayed stable around $550-600 \mathrm{~km} / \mathrm{s}$. Figure $1 \mathrm{~d}$ and $1 \mathrm{e}$ show the $\mathrm{H}^{+}$density and energy flux, respectively. The trajectory of each orbit is shown in Figure 2i. In Orbit 839, the solar wind was shocked with increased solar wind flux and strengthened IMF. The solar wind flux and density increased gradually to a peak at 04:00 UT on 7 March, and then decreased. It is hard to identify the arrival time of the ICME shock and ICME body, but Mars responded drastically in Orbit 840, which should correspond to the strongest part of the solar storm. The ICME with stronger pressure should impact the Martian upper atmosphere strongly and induce a series of planetary responses.

\subsection{Pick-up $\mathrm{H}^{+}$Enhancement}

As the solar wind approaches Mars, newly formed planetary ions are picked up by the solar wind convection electric field. This pickup process, in which momentum and energy are transferred from solar wind to local plasma, may accelerate these ions to the escape velocity (Bertucci et al., 2011). Because of the low energy of partially picked up $\mathrm{H}^{+}$compared to solar wind ( $\left.1 \mathrm{keV}\right)$, some pickup protons buried under solar wind fluxes are difficult to interpret from the angle-averaged spectrum (Rahmati et al., 2017). One objective of SWIA is to determine the pick-up acceleration of planetary-origin ions (Halekas et al., 2015a). Based on directional analysis of SWIA Coarse data proposed by Rahmati et al. (2017), choice of a specific direction from which solar wind protons are at a minimum should allow pick-up $\mathrm{H}^{+}$to be distinguished from background ions.

Figure 2 displays the comparison of measured pick-up fluxes in quiet solar wind and under the impact of the ICME. Figure $2 \mathrm{~g}$ shows the differential energy flux in one direction $\left(22.5^{\circ} \times 22.5^{\circ}\right.$, labeled AOD1) of SWIA anodes before the arrival of the ICME, and Figure $2 \mathrm{~h}$ shows the fluxes measured by the same anode (AOD1) during the ICME. Since MAVEN is a three-axis stabilized spacecraft, over time the same anodes indicate the same observational directions. The pick-up $\mathrm{H}^{+}$fluxes exhibit a discontinuous spectrum at about $4 \mathrm{keV}$. Due to the small field of view (FOV) of each bin of SWIA, even a slight change in the direction of the IMF can significantly alter the pick-up ion fluxes in the FOV of each bin (Rahmati et al., 2017). The greatly disturbed pick-up fluxes on 7 March are most probably due to the unstable IMF strength and direction during the ICME passage. As marked by the golden color in Figure $2 \mathrm{i}$, the observations of pre-ICME and during-ICME orbits are both at the same range of altitudes upstream of the bow shock. Compared with Figure $2 \mathrm{~g}$, the pickup $\mathrm{H}^{+}$fluxes show higher values in Figure $2 \mathrm{~h}$. In addition, the measurements from all 64 anodes were checked in Orbits 837 and 840 and several anodes observed evidence of pick-up $\mathrm{H}^{+}$(not shown). The pick-up $\mathrm{H}^{+}$fluxes in these anodes were significantly enhanced during the passage of the ICME.

The enhancement of pick-up $\mathrm{H}^{+}$flux is caused by an increase in 
(a)

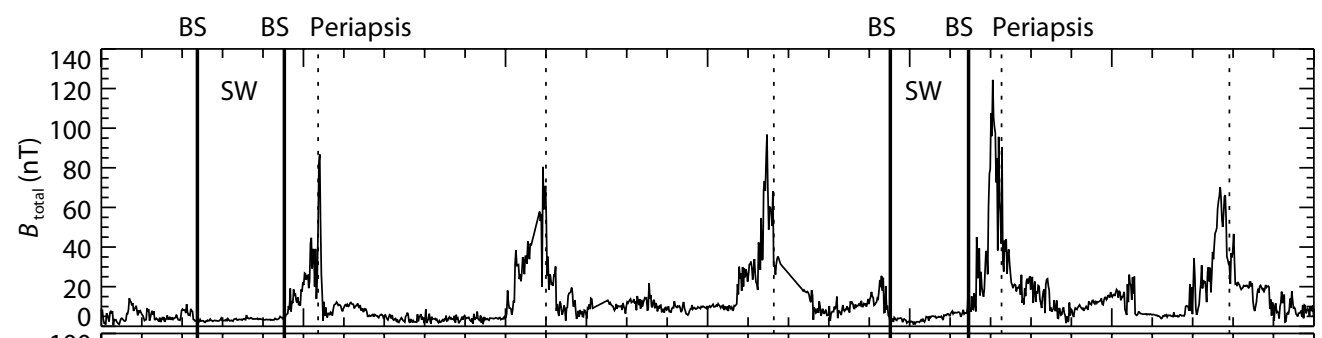

(b)

(c)

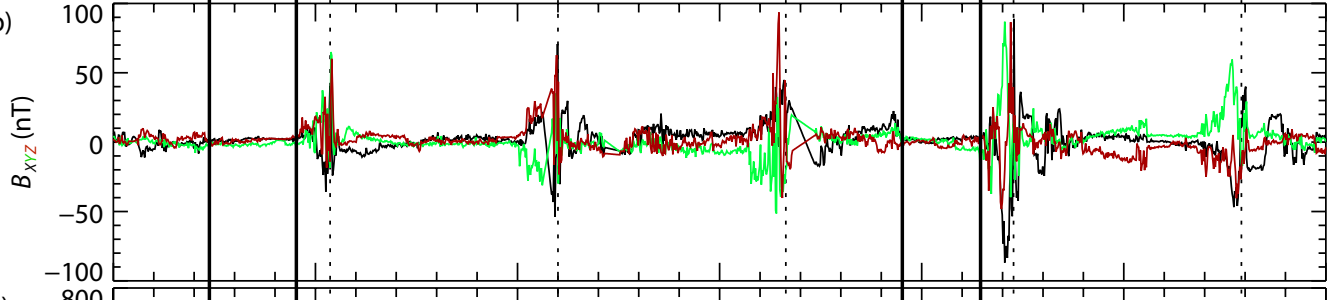

(d)
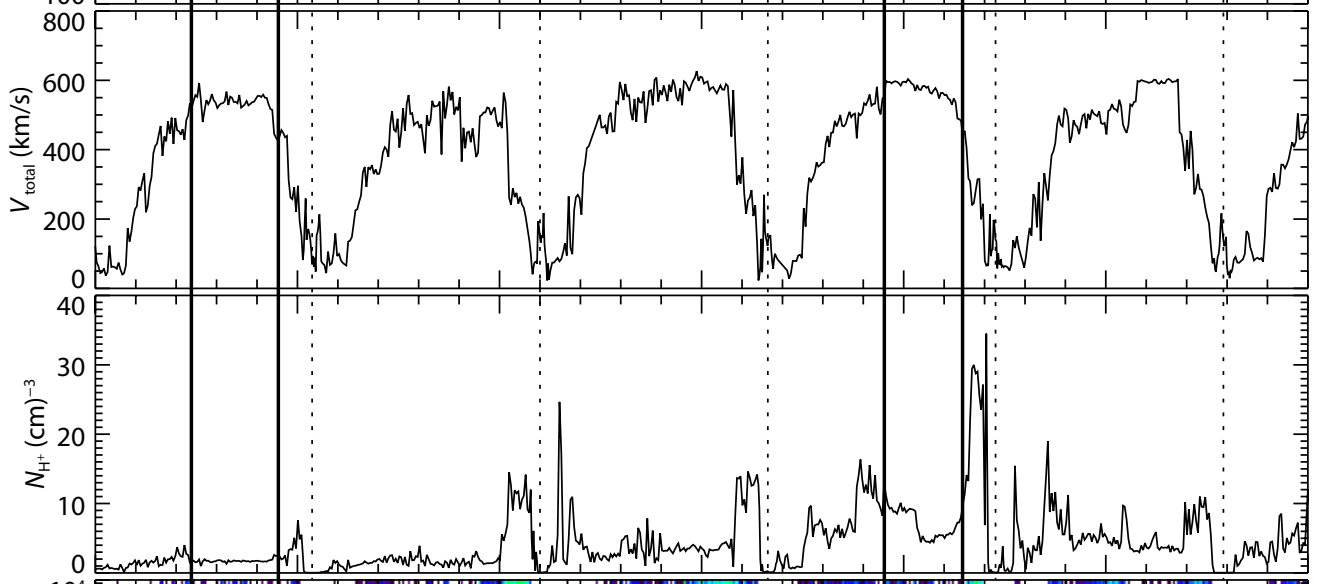

(e)

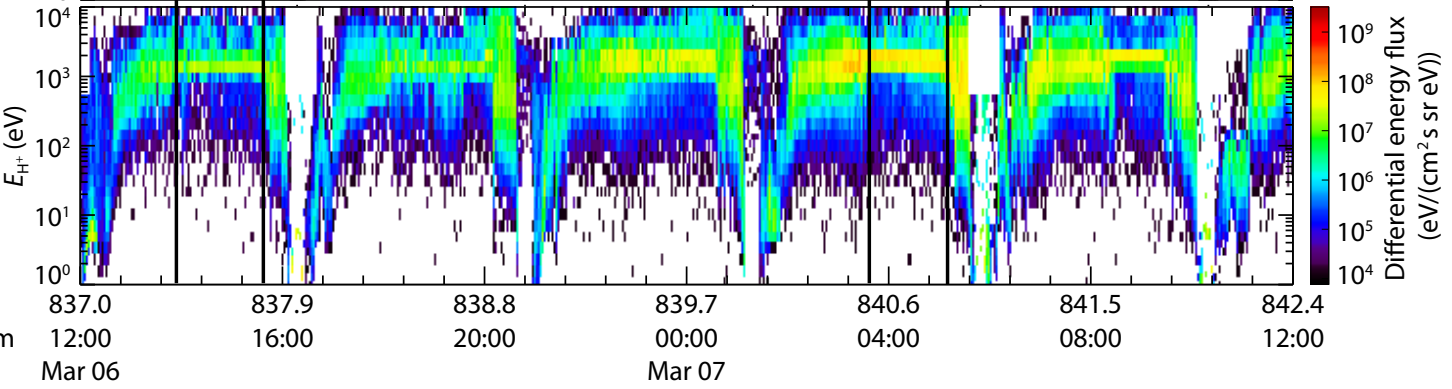

Figure 1. Overview of the ICME on 7 March 2015 observed by MAVEN. (a) The magnetic field strength. (b) Three components of magnetic field in MSO coordinates. (c) The ion velocity. (d) The $\mathrm{H}^{+}$density. (e) The differential energy flux of $\mathrm{H}^{+}$. Solid vertical lines denote the bow shock crossings in orbit 837 and 840 and vertical dash lines denote the periapsis crossings. Note that all the data are smoothed with a time width of 60 seconds.

the rate of production of exospheric protons. The ionization rate is determined mainly by charge-exchange reactions rather than by EUV radiation (Rahmati et al., 2018). The production rate of exospheric $\mathrm{H}^{+}$due to the charge-exchange reaction depends on the solar wind flux and local neutral $\mathrm{H}$ density: $p\left(\mathrm{~cm}^{-3} \mathrm{~s}^{-1}\right)=$ $\sigma_{\mathrm{CX}}\left(\mathrm{cm}^{2}\right) \times N_{\mathrm{SW}}\left(\mathrm{cm}^{-3}\right) \times V_{\mathrm{SW}}(\mathrm{cm} / \mathrm{s}) \times N_{0}\left(\mathrm{~cm}^{-3}\right)$, where $p$ is the production rate of exospheric $\mathrm{H}^{+}, \sigma_{\mathrm{CX}}$ is the cross section, $N_{S W}$ is the solar wind density, $V_{S W}$ is the solar wind velocity, and $N_{0}$ is the local neutral $\mathrm{H}$ density. According to Figure 2, the averaged solar wind densities observed in Orbit 837 (14:30-15:30 UT on 6 March) and 840 (04:00-05:00 UT on 7 March) are $1.7 \mathrm{~cm}^{-3}$ and $6.1 \mathrm{~cm}^{-3}$, respectively, while the solar wind velocities are $540 \mathrm{~km} / \mathrm{s}$ and 571 $\mathrm{km} / \mathrm{s}$, respectively. The increase of solar wind flux might be one reason for increased pick-up $\mathrm{H}^{+}$. But the total increased pick-up $\mathrm{H}^{+}$ flux and the contribution of background neutral $\mathrm{H}$ density are hard to estimate quantitatively due to the turbulent IMF. A newborn $\mathrm{H}^{+}$by charge exchange is accompanied by a new energetic neutral atom (ENA). The background neutral hydrogen can be estimated better by ENAs since the measurements of ENA are not affected by variations in the IMF direction.

\subsection{Penetrating $\mathbf{H}^{+}$Enhancement}

The hydrogen corona can spread as far as 10 Mars radii upstream of the bow shock (Chaffin et al., 2015). Charge exchange could happen directly between solar wind protons and exospheric neutral hydrogen atoms. As electrons are captured by solar wind protons, ENAs are formed with the same velocity as the solar wind (Kallio et al., 1997). These ENAs can penetrate into Mars' atmosphere without the obstructions of IMF and bow shock. In this way, the solar wind deposits energy into the atmosphere. ENAs 

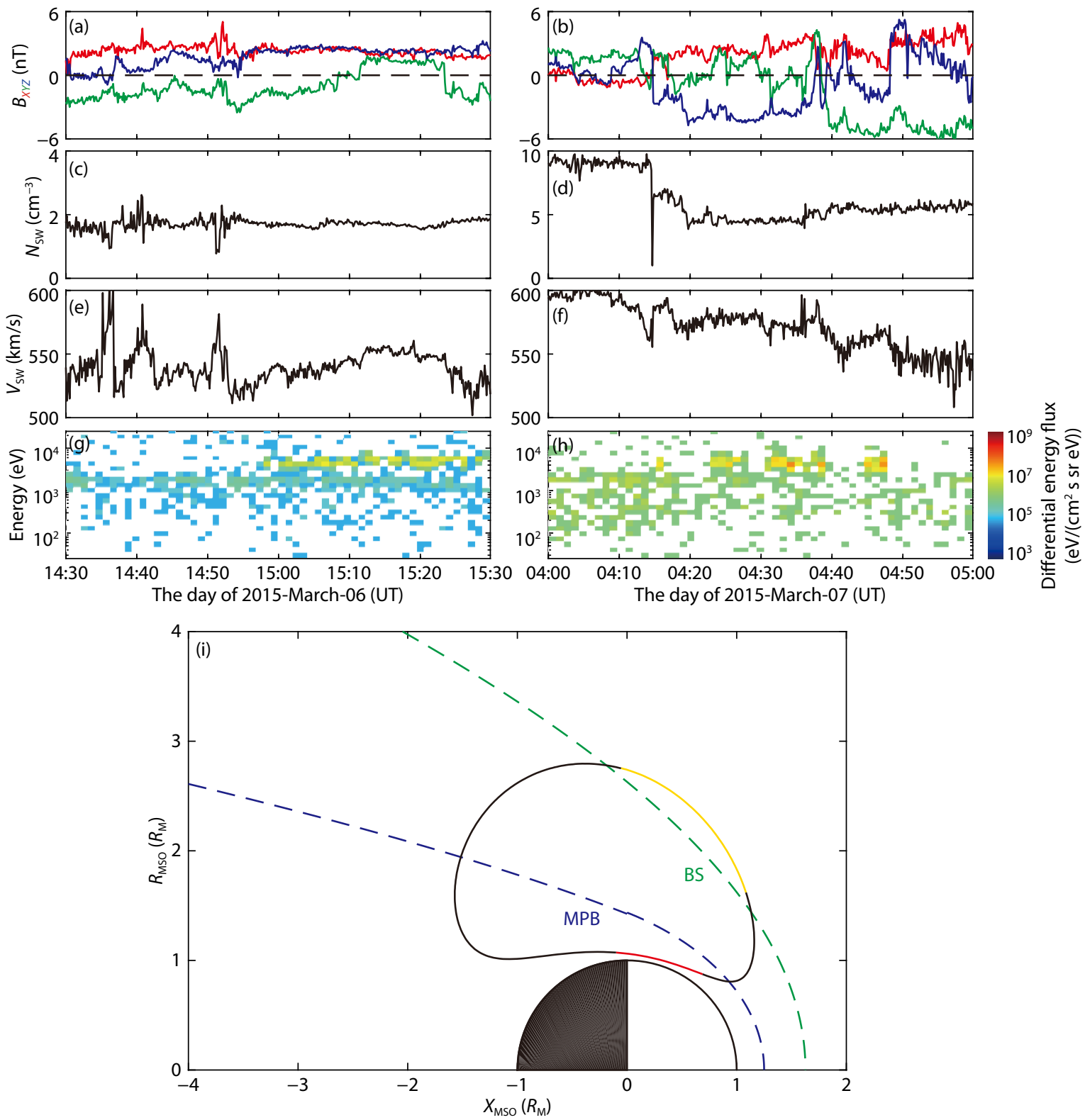

Figure 2. The pick-up $\mathrm{H}^{+}$fluxes measured by SWIA during the passage of ICME compared with that in quiet solar wind. (a)-(b) The three components of magnetic field in MSO coordinates. (c)-(d) Solar wind density. (e)-(f) Solar wind velocity. (g)-(h) The differential energy flux of pick-up $\mathrm{H}^{+}$recorded by anode A0D1 of SWIA. (i) The trajectory of each orbit from 6 March to 7 March. The spacecraft position is marked by golden color when measuring pick-up flux and red color when measuring penetrating $\mathrm{H}^{+}$. The dashed lines are the bow shock (BS) location and magnetic pileup boundary (MPB) modeled by Trotignon et al. (2006).

will collide with atmospheric particles and lead to ionizations. The energetic ionospheric protons resulting from the impact of these ENAs with planetary particles at lower altitudes are termed "penetrating $\mathrm{H}^{+"}$ (Halekas, 2017). Penetrating $\mathrm{H}^{+}$can be observed by SWIA in the dayside atmosphere. These $\mathrm{H}^{+}$populations have the same energy and velocity as the upstream solar wind (Halekas et al., 2015b). Figure 3 shows penetrating $\mathrm{H}^{+}$observations in the near-periapsis region before and after the arrival of the ICME. The solar wind is obvious at approximately $1.5 \mathrm{keV}$ in the spectra. As the ICME passed by, the solar wind fluxes were enhanced compared with those on the day before. The penetrating protons are indicated by the dashed boxes, showing long, narrow and tenuous spectra with the same energy as the solar wind. Since penet- rating protons retain the velocity and thus the energy of the solar wind, the density of penetrating $\mathrm{H}^{+}$can be indicated by its flux. It is clear that the penetrating $\mathrm{H}^{+}$flux and density increased greatly during the passage of the ICME. In Figure 4, the flux spectra are integrated over periods when MAVEN stayed near the periapsis. The penetrating $\mathrm{H}^{+}$flux concentrates around $1.5 \mathrm{keV}$ as marked by the vertical dashed lines in Figure 3. Note that the second peak of penetrating $\mathrm{H}^{+}$flux at $5 \mathrm{keV}$ on 6 March is not real, which can be seen from Figure $3 \mathrm{~b}$. The counts of integrated flux increased from about 4000 to 20000 and the average density of the penetrating $\mathrm{H}^{+}$increased accordingly by a factor of 5 .

Penetrating protons contain information about the hydrogen 

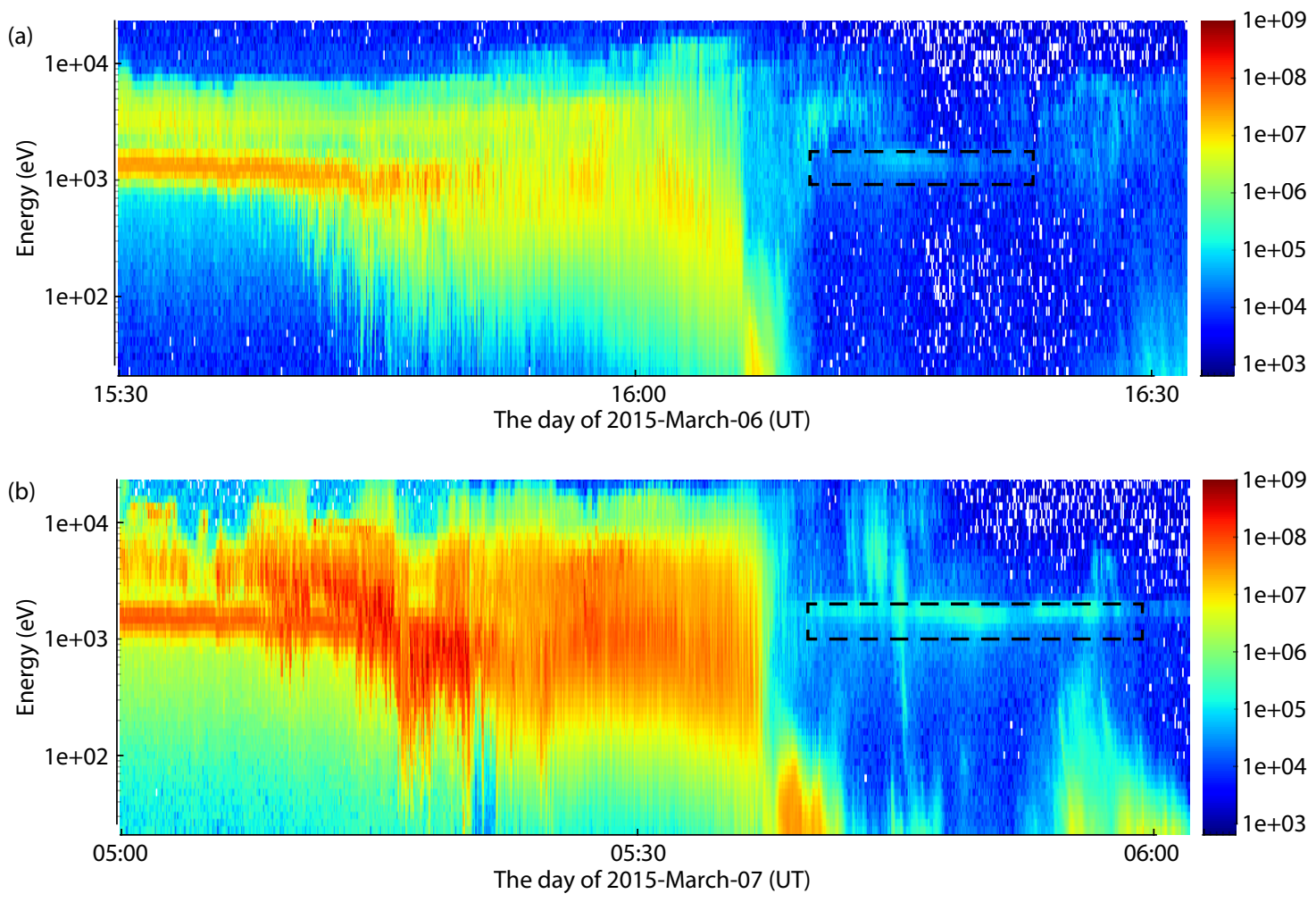

Figure 3. The SWIA observation of penetrating proton during the ICME compared with that in quiet solar wind. The angle-averaged differential energy flux spectra (a) before the arrival of the ICME and (b) during the passage of the ICME. The energy of the solar wind is at approximately 1.5 $\mathrm{keV}$. The penetrating protons are bounded by black dashed boxes. In comparison, the penetrating proton fluxes increased during the ICME on 7 March.

corona. However, the estimated density of penetrating $\mathrm{H}^{+}$by MAVEN observations varies with orbit altitude, solar wind parameters, and the $\mathrm{H}$ corona (Halekas, 2017). As shown in Figure $2 \mathrm{i}$ and Figure 3, the measurements before and during the ICME are made at almost the same altitude near the periapsis. The enhancement of penetrating $\mathrm{H}^{+}$density is therefore attributed to the change of solar wind and the change of the $\mathrm{H}$ corona. To infer the properties of the $\mathrm{H}$ corona, an equation was suggested by Halekas (2017): $N_{\text {pen }}(r)=N_{\mathrm{SW}} \times \sigma_{\mathrm{H}}\left(V_{\mathrm{SW}}\right) \times C_{\exp }\left(R_{\mathrm{BS}}\right) \times F^{+}\left(r ; V_{\mathrm{SW}}\right)$, where $N_{\text {pen }}$ is the penetrating $\mathrm{H}^{+}$density, $r$ is the radius, $N_{S W}$ is the solar wind density, $\sigma_{\mathrm{H}}$ is the cross section, $V_{\mathrm{SW}}$ is the solar wind velocity, $C_{\text {exp }}$ is the exospheric column density upstream from the bow shock, $R_{\mathrm{BS}}$ is the bow shock position, and $F^{+}$is the fraction of precipitating ENAs converted to positively charged hydrogen.

The spacecraft crossed the bow shock at 15:44 UT on 6 March and at 05:13 UT on 7 March. The averaged solar wind density and velocity during quiet solar wind (15:00-15:30 UT on 6 March) are 1.70 $\mathrm{cm}^{-3}$ and $541 \mathrm{~km} / \mathrm{s}$, respectively. And the averaged solar wind density and velocity in the ICME (04:30-05:00 UT on 7 March) are $5.25 \mathrm{~cm}^{-3}$ and $558 \mathrm{~km} / \mathrm{s}$, respectively. The penetrating $\mathrm{H}^{+}$density $\left(N_{\text {pen }}\right)$ at radius $r$ increased by a factor of 5 , while the solar wind density $\left(N_{\mathrm{SW}}\right)$ increased by a factor of 3 . The cross section $\left(\sigma_{\mathrm{H}}\right)$ should not change significantly because the solar wind velocity $\left(V_{\text {SW }}\right)$ remained roughly constant. The fraction of precipitating ENAs converted to positively charged hydrogen $\left(F^{+}\right)$is given by $F^{+}=\sigma_{01}\left(V_{S W}\right) /\left(\sigma_{01}\left(V_{S W}\right)+\sigma_{10}\left(V_{S W}\right)\right)$, where $\sigma_{01}\left(V_{S W}\right)$ is the electron stripping cross section and $\sigma_{10}\left(V_{S W}\right)$ is the charge exchange cross section (Halekas, 2017). Based on the cross sections from Lindsay et al. (2005), the increase in solar wind velocity from 541 $\mathrm{km} / \mathrm{s}$ to $558 \mathrm{~km} / \mathrm{s}$ would not lead to significant change of $\mathrm{F}^{+}$. Consequently, the increase in penetrating $\mathrm{H}^{+}$density resulted in part from the increase in exospheric column density upstream from the bow shock $\left(C_{\text {exp }}\right)$. Bow shock positions $\left(R_{\mathrm{BS}}\right)$ can be identified by the sudden jump in magnetic field strength and the cessation of stable solar wind flux, as shown in Figure 1. By checking the position of the spacecraft when crossing the bow shock (not shown here), the observed bow shock location moved from $1.625 R_{\mathrm{M}}$ (radius of Mars) in the quiet solar wind to $1.69 R_{\mathrm{M}}$ under the impact of the ICME. Such a small difference can be basically neglected. From the quantitative estimation above, the increment of $C_{\exp }$ strongly indicates a denser hydrogen corona during the passage of the ICME.

\section{Discussions and Conclusions}

This paper reports observations of the pick-up $\mathrm{H}^{+}$and penetrating $\mathrm{H}^{+}$during the passing of the ICME on 7 March 2015. We infer from these observations that the ICME increased the exospheric $\mathrm{H}$ density upstream of the bow shock. The fluxes of pick-up $\mathrm{H}^{+}$ measured in the ICME are observed to have been enhanced. The fluxes of penetrating $\mathrm{H}^{+}$with the same energy as the solar wind in the ionosphere increased evidently during the ICME event. Our analysis indicates that the total flux of these $\mathrm{H}^{+}$increased by a factor of approximately 5 . According to the model proposed by Halekas (2017), the estimated column density upstream of the 

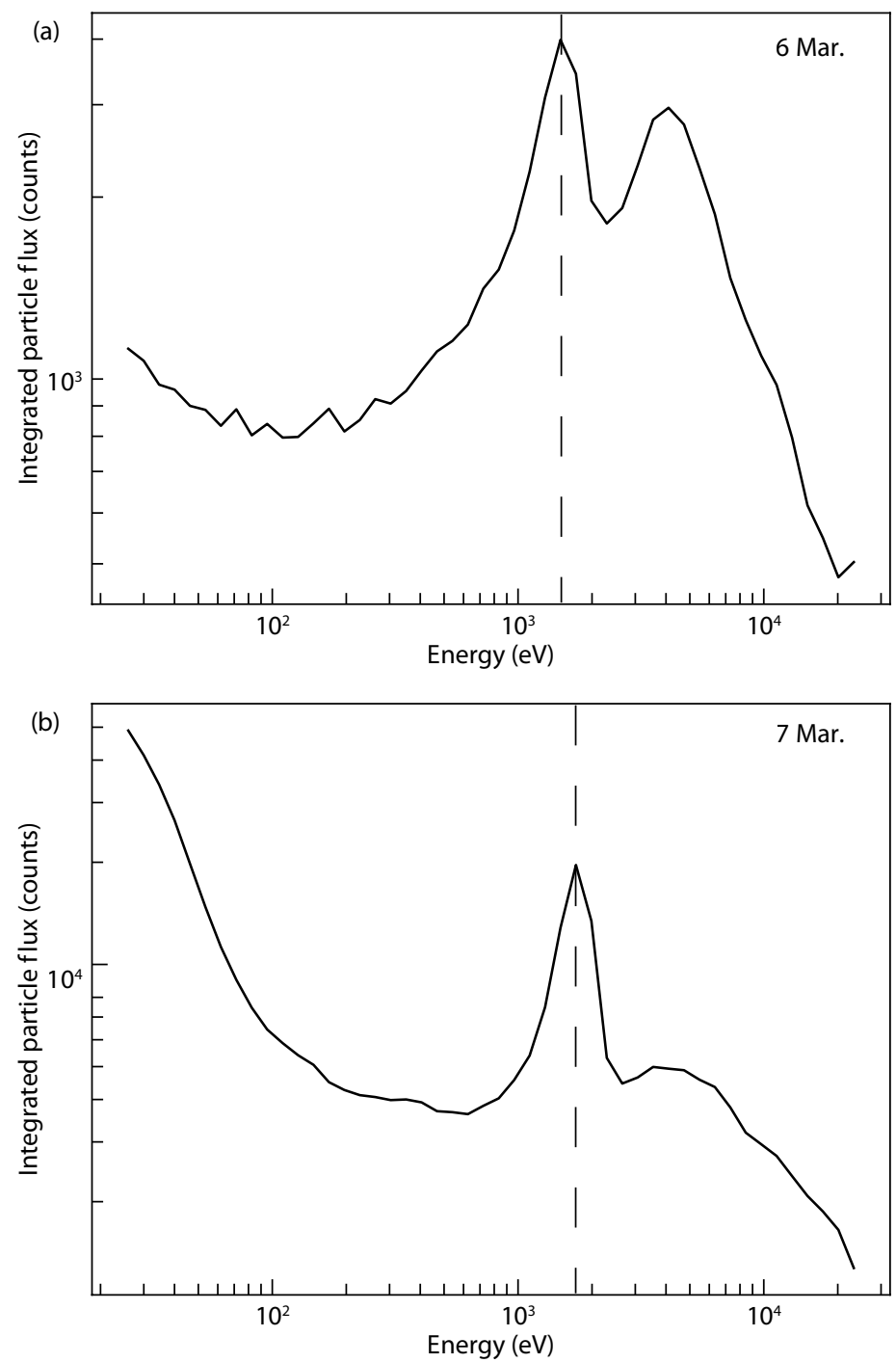

Figure 4. The integrated penetrating $\mathrm{H}^{+}$flux near the periapsis (a) in the pre-ICME orbit (16:10-16:25 UT on 6 March) and (b) during ICME orbit (05:40-05:55 UT on 7 March). The position of spacecraft when taking the 15-minutes measurements is marked by red color in Figure 2i. The energy of penetrating $\mathrm{H}^{+}$is marked by vertical dash lines.

bow shock increased. In other words, the neutral hydrogen density upstream of the bow shock increased under the impact of the ICME including the accompanying SEPs.

The increase of hydrogen density might not be coming from the supply in lower atmosphere, because the upward transport of hydrogen has a much longer timescale. The upward transport of hydrogen from the planetary surface is a relatively slow process (McElroy and Donahue, 1972). Dust storm impact could be ruled out since the enhanced hydrogen escape during global dust storms is caused by changes in high-altitude water vapor (Heavens et al., 2018), which plays a role on a timescale of weeks (Chaffin et al., 2017). Since the total abundance of exospheric $\mathrm{H}$ should not be disturbed significantly in such a short time (hours), we propose that the $\mathrm{H}$ exosphere itself expanded significantly, providing more $\mathrm{H}$ atoms to the outer exosphere. The expansion might result from solar energy deposits during the solar storm. The impact of an ICME, which is characterized by stronger fluxes, magnetic field, and dynamic pressure, can induce intense responses from Mars. The solar wind interaction with Mars can be greatly strengthened during an ICME encounter (Jakosky et al., 2015a). The enhancements of ionospheric magnetic fields and electron densities during an ICME are attributed to the high dynamic pressure and high SEP flux (Harada et al., 2018). The ionosphere/exosphere can be excited and heated during an ICME. Note that the solar flare influence can be excluded because the recovery timescale is relatively short and the latest solar flare was more than 5 MAVEN orbits before the event. With increased energy, more $\mathrm{H}$ atoms in ballistic motion can reach a distance greater than several Mars radii, producing a denser outer $\mathrm{H}$ corona upstream of the bow shock. This result is consistent with a previous study, which reported that the density of hydrogen at the exobase does not increase during an ICME but that the neutral temperature can be enhanced (Mayyasi et al., 2018).

The ICME which impacted Mars on 7 March 2015 led to a denser outer hydrogen corona. It is important to point out that our conclusion is based on the assumption that solar wind parameters remained effectively unchanged when MAVEN was in the induced magnetosphere. Without measurements of the hydrogen density 
and temperature at the exobase, the Jeans escape rate cannot be estimated quantitatively. But the expansion of the exosphere strongly suggests a heating effect caused by the ICME impact. The excited and heated hydrogen exosphere during the ICME probably indicates an elevated hydrogen escape rate.

\section{Acknowledgments}

This work is funded by the Science and Technology Development Fund, Macao SAR (File no. 0002/2019/A1) and National Natural Science Foundation of China (NSFC) under grant 41731067. We acknowledge the MAVEN contract for support. The data for this study are available at MAVEN science data center (https://lasp.colorado.edu/maven/sdc/public/) and the AMDA (http://amda. cdpp.eu/) science analysis system provided by the Centre de Données de la Physique des Plasmas (CDPP) supported by CNRS, CNES, Observatoire de Paris and Université Paul Sabatier, Toulouse.

\section{References}

Anderson, Jr. D. E., and Hord, C. W. (1971). Mariner 6 and 7 ultraviolet spectrometer experiment: Analysis of hydrogen Lyman-alpha data. J. Geophys. Res., 76(28), 6666-6673. https://doi.org/10.1029/JA076i028p06666

Bertucci, C., Duru, F., Edberg, N., Fraenz, M., Martinecz, C., Szego, K., and Vaisberg, O. (2011). The induced magnetospheres of Mars, Venus, and Titan. Space Sci. Rev., 162(1-4), 113-171. https://doi.org/10.1007/s11214-011-98451

Bhattacharyya, D., Clarke, J. T., Bertaux, J. L., Chaufray, J. Y., and Mayyasi, M. (2015). A strong seasonal dependence in the Martian hydrogen exosphere. Geophys. Res. Lett., 42(20), 8678-8685. https://doi.org/10.1002/2015GL065804

Chaffin, M. S., Chaufray, J. Y., Deighan, J., Schneider, N. M., McClintock, W. E., Stewart, A. I. F., Thiemann, E., Clarke, J. T., Holsclaw, G. M., ... Jakosky, B. M. (2015). Three-dimensional structure in the Mars $\mathrm{H}$ corona revealed by IUVS on MAVEN. Geophys. Res. Lett., 42(21), 9001-9008.

https://doi.org/10.1002/2015GL065287

Chaffin, M. S., Deighan, J., Schneider, N. M., and Stewart, A. I. F. (2017). Elevated atmospheric escape of atomic hydrogen from Mars induced by highaltitude water. Nat. Geosci., 10(3), 174-178. https://doi.org/10.1038/ngeo2887

Chaufray, J. Y., Bertaux, J. L., Leblanc, F., and Quémerais, E. (2008). Observation of the hydrogen corona with SPICAM on Mars Express. Icarus, 195(2), 598-613. https://doi.org/10.1016/j.icarus.2008.01.009

Chaufray, J. Y., Gonzalez-Galindo, F., Forget, F., Lopez-Valverde, M. A., Leblanc, F., Modolo, R., and Hess, S. (2015). Variability of the hydrogen in the Martian upper atmosphere as simulated by a 3D atmosphere-exosphere coupling. Icarus, 245, 282-294. https://doi.org/10.1016/j.icarus.2014.08.038

Curry, S. M., Luhmann, J. G., Ma, Y. J., Dong, C. F., Brain, D., Leblanc, F., Modolo, R., Dong, Y., McFadden, J., ... Jakosky, B. (2015). Response of Mars O+ pickup ions to the 8 March 2015 ICME: Inferences from MAVEN data-based models. Geophys. Res. Lett., 42(21), 9095-9102. https://doi.org/10.1002/2015GL065304

Halekas, J. S., Taylor, E. R., Dalton, G., Johnson, G., Curtis, D. W., McFadden, J. P., Mitchell, D. L., Lin, R. P., and Jakosky, B. M. (2015a). The solar wind ion analyzer for MAVEN. Space Sci. Rev., 195(1-4), 125-151. https://doi.org/10.1007/s11214-013-0029-z

Halekas, J. S., Lillis, R. J., Mitchell, D. L., Cravens, T. E., Mazelle, C., Connerney, J. E. P., Espley, J. R., Mahaffy, P. R., Benna, M., ... Ruhunusiri, S. (2015b). Maven observations of solar wind hydrogen deposition in the atmosphere of Mars. Geophys. Res. Lett., 42(21), 8901-8909.

https://doi.org/10.1002/2015GL064693

Halekas, J. S. (2017). Seasonal variability of the hydrogen exosphere of Mars. J.
Geophys. Res.: Planets, 122(5), 901-911. https://doi.org/10.1002/2017JE005306

Harada, Y., Gurnett, D. A., Kopf, A. J., Halekas, J. S., Ruhunusiri, S., DiBraccio, G. A., Espley, J. D., and Brain, A. (2018). MARSIS observations of the Martian nightside ionosphere during the September 2017 solar event. Geophys. Res. Lett., 45(16), 7960-7967. https://doi.org/10.1002/2018GL077622

Heavens, N. G., Kleinböhl, A., Chaffin, M. S., Halekas, J. S., Kass, D. M., Hayne, P. O., McCleese, D. J., Piqueux, S., Shirley, J. H., and Schofield, J. T. (2018). Hydrogen escape from Mars enhanced by deep convection in dust storms. Nat. Astron., 2(2), 126-132. https://doi.org/10.1038/s41550-017-0353-4 Jakosky, B. M., Grebowsky, J. M., Luhmann, J. G., Connerney, J., Eparvier, F., Ergun, R., Halekas, J., Larson, D., Mahaffy, P., ... Yelle, R. (2015a). Maven observations of the response of Mars to an interplanetary coronal mass ejection. Science, 350(6261), aad0210.

https://doi.org/10.1126/science.aad0210

Jakosky, B. M., Lin, R. P., Grebowsky, J. M., Luhmann, J. G., Mitchell, D. F., Beutelschies, G., Priser, T., Acuna, M., Andersson L., ... Zurek, R. (2015b). The Mars atmosphere and volatile evolution (MAVEN) mission. Space Sci. Rev., 195(1-4), 3-48. https://doi.org/10.1007/s11214-015-0139-x

Jakosky, B. M., Brain, D., Chaffin, M., Curry, S., Deighan, J., Grebowsky, J., Halekas, J., Leblanc, F., Lillis, R., ... Zurek, R. (2018). Loss of the Martian atmosphere to space: Present-day loss rates determined from MAVEN observations and integrated loss through time. Icarus, 315, 146-157. https://doi.org/10.1016/j.icarus.2018.05.030

Kallio, E., Luhmann, J. G., and Barabash, S. (1997). Charge exchange near Mars: The solar wind absorption and energetic neutral atom production. J. Geophys. Res.: Space Phys., 102(A10), 22183-22197. https://doi.org/10.1029/97JA01662

Lee, C. O., Hara, T., Halekas, J. S., Thiemann, E., Chamberlin, P., Eparvier, F., Lillis, R. J., Larson, D. E., Dunn, P. A., ... Jakosky, B. M. (2017). MAVEN observations of the solar cycle 24 space weather conditions at Mars. J. Geophys. Res.: Space Phys., 122(3), 2768-2794. https://doi.org/10.1002/2016JA023495

Lindsay, B. G., Yu, W. S., and Stebbings, R. F. (2005). Cross sections for chargechanging processes involving kilo-electron-volt $\mathrm{H}$ and $\mathrm{H}^{+}$with $\mathrm{CO}$ and $\mathrm{CO}_{2}$. Phys. Rev. A, 71(3), 032705. https://doi.org/10.1103/PhysRevA.71.032705

Mannucci, A. J., Tsurutani, B. T., lijima, B. A., Komjathy, A., Saito, A., Gonzalez, W. D., Guarnieri, F. L., Kozyra, J. U., and Skoug, R. (2005). Dayside global ionospheric response to the major interplanetary events of October 29-30, 2003 "Halloween Storms". Geophys. Res. Lett., 32(12), L12S02. https://doi.org/10.1029/2004GL021467

Mayyasi, M., Bhattacharyya, D., Clarke, J., Catalano, A., Benna, M., Mahaffy, P., Thiemann, E., Lee, C. O., Deighan, J., ... Jakosky, B. (2018). Significant space weather impact on the escape of hydrogen from Mars. Geophys. Res. Lett., 45(17), 8844-8852. https://doi.org/10.1029/2018GL077727

McElroy, M. B., and Donahue, T. M. (1972). Stability of the Martian atmosphere. Science, 177(4053), 986-988. https://doi.org/10.1126/science.177.4053.986

Rahmati, A., Larson, D. E., Cravens, T. E., Lillis, R. J., Halekas, J. S., McFadden, J. P., Dunn, P. A., Mitchell, D. L., Thiemann, E. M. B., ... Jakosky, B. M. (2017). MAVEN measured oxygen and hydrogen pickup ions: Probing the Martian exosphere and neutral escape. J. Geophys. Res.: Space Phys., 122(3), 3689-3706. https://doi.org/10.1002/2016JA023371

Rahmati, A., Larson, D. E., Cravens, T. E., Lillis, R. J., Halekas, J. S., McFadden, J. P., Mitchell, D. L., Thiemann, E. M. B., Connerney, J. E. P., ... Jakosky, B. M. (2018). Seasonal variability of neutral escape from Mars as derived from MAVEN pickup ion observations. J. Geophys. Res.: Planets, 123(5), 1192-1202. https://doi.org/10.1029/2018JE005560

Trotignon, J. G., Mazelle, C., Bertucci, C., and Acuña, M. H. (2006). Martian shock and magnetic pile-up boundary positions and shapes determined from the Phobos 2 and Mars Global Surveyor data sets. Planet. Space Sci., 54(4), 357-369. https://doi.org/10.1016/j.pss.2006.01.003

Xu, Q., Xu, X. J., Chang, Q., Rong, Z. J., Wang, J., Xu, J. Y., and Zhang, T. L. (2019). Observations of the Venus dramatic response to an extremely strong Interplanetary Coronal Mass Ejection. Astrophys. J., 876(1), 84. https://doi.org/10.3847/1538-4357/ab14e1 